\title{
Reproductibilitéde la correction d'une épreuve de lecture critique d'article : évaluation par une étude pilote chez 59 étudiants en médecine
}

\author{
Francis ROUSSE ', Pierre CZERNCHOW', Alain LAVONNE', \\ Jean-François $L E M E A N D^{4}$, Jean-Paul RLLASTRE ${ }^{5}$
}

\begin{abstract}
Résumé Contexte : L'introduction d'uneépreuve de lecturecritique d'artides dansl'examen classant national en 2008 implique de former les étudiants et de mettre au point une procédure reproductible de correction. Actuellement la formation est très variable entre les différentes facultés et la reproductibilité de ce type d'épreuve est inconnue. But : Evaluer la reproductibilité de la correction d'une épreuve de lecture critique d'artides dans des conditions proches de celles del'examen national dassant. Sujets et méthodes : 59 étudi ants volontaires de $2^{\mathrm{e}}$ et $3^{\mathrm{e}}$ an nées du deuxième cyde des études médicales ont passé une épreuve surveillée de trois heures, sur un artide original en françaiş comportant 10 questionsvalidées et un résumé de 250 mots au plus Trois enseignants ayant préparé le dosser de l'épreuve ont corrigé en aveugle les questions et le résumés sol 16 items, sans grille de correction. L'accord entre les troi spaires de correcteurs a étéapprécié par les coefficients de corrélation intra dasse $(\mathrm{CCl})$. Résultats : En moyenne, l'accord entre les correcteurs était modéré ou médiocre: $\mathrm{CCI}=$ $0,68$ (intervalle de confiance à $95 \%: 0,52-0,80), \mathrm{CCl}=0,71(0,55-0,81)$ et $\mathrm{CCl}=0,48(0,26-0,65)$ selon la paire Cette concordance était variable suivant les items (très mauvaise pour deux questions). Elle était mé lleure pour les questions que pour le résumé pour 2 des 3 paires de correcteurs. Conclusi on : Cette méthode de correction de l'épreuve de lecture critique d'articles est peu reproductible. L'impréparation actuelle des étudiants et des enseignants est probablement un facteur aggravant.
\end{abstract}

Mot Clés Lecture critique d'articles; évaluation ; reproductibilité inter correcteurs ; examen classant national.

Abstract Context: At french medical univerities, a new national sorting exam (N SE) has been implemented, which will includefrom 2008 onwardsa test of scientific papers critical analysis(SPCA). This impliesfurther educational courses for studentsand a reliablemarking process Currently, educational development ishighly variable between universities, and the reliability of the marking process remains unknown. Aim: To evaluate the reliability of SPCA test marking, in conditions close to those of the N SE. Subjects and methods. 59 volunteer medical students from 4th or 5 th year at the Rouen university medical school completed a supervised examination of three hours, on an original artide in French, induding 10 validated open questions and an abstract of less than 250 words. Three teachers, who prepared the assessment, blindly marked the questions and the abstract, according to 16 items, without a correction grid. The agreement among the three pairs of correctors was evaluated according to the intradass correlation coefficients (ICC). Results. The overall mean ICCS (95\% confidence interval) for the 3 pairs were moderate or poor: $0.68(0.52-0.80), 0.71(0.55-0.81)$ and $0.48(0.26$ -0.65 ). The marking agreement was better for the questionsthan for the abstract for 2 out of the 3 pairs of teachers. It was variable with the items, with a very poor score for two of them. Conclusion: The reliability of this SPCA test marking method was considered poor. The present lack of sudents and faculty preparation is probably a worsening factor.

Key words papers critical analysis; assessment; reliability; national ranking exam.

Pédagogie M édicale 2005; 6: 71-78

1- Département d'Anatomie et de Cytologie Pathologiques - Faculté de Médecine - 76000 Rouen - France

2- Département d'Epidémiologie et de santé publique ; même adresse

3- Laboratoire de Biochimie; même adresse

4- Département de Microbiologie; même adresse

5- Service de Néphrologie ; même adresse

Correspondance: Francis Roussel - Pavillon Jacques Delarue - CHU de Rouen - 76031 Rouen Cedex - France

Tél.: 0232888067 - mailto: Francis.Roussel@chu-rouen.fr 


\section{Recherche et Perspectives}

\section{Introduction}

La formation continue des médecins implique la lecture régulière de travaux scientifiques ; les médecins doivent, en effet, pouvoir mettre à jour leur documentation professionnelle, ce qui implique en particulier d'analyser et juger de façon autonome la valeur scientifique des articles publiés, ainsi que leur caractère applicable à d'autres situations.

L'enseignement de la lecture critique d'article (LCA) vise à compléter leur formation initiale dans ce domaine ; elle substitue partiellement l'acquisition d'un savoir (connaissances) par celle d'un savoir-faire (sélectionner soi-même des connaissances valides).

Cet enseignement, qui doit préparer les futurs médecinsà leur formation continue, est différent de l'expertise scientifique requise en amont, pour sélectionner un manuscrit en vue d'une éventuelle publication scientifique ${ }^{1}$ (processus éditorial).

\section{Contexte}

Une épreuve de LCA des étudiants est prévue dans le cadre de l'examen classant national (ECN) à partir de $2008^{2}$, selon des modalités définies par le Centre national du concours de l'internat $(\mathrm{CNCl})$. Cette nouvelle épreuve de trois heures, comptant pour $20 \%$ des points, devrait concerner un article original, de portée générale, écrit en langue française ; elle devrait associer six à dix questions sur la méthodologie du travail, d'une part, et la rédaction d'un résumé de moins de 250 mots, d'autre part.

La plupart des facultés de médecine ont mis en place de façon empirique dès 2002 des formations à cetteépreuve ; toutefois, ni les étudiants, ni les correcteurs ne sont entrânés à de telles épreuves nouvelles, alors qu'une telle expé rience est nécessaire pour évaluer l'acquisition ${ }^{3}$ de cette habileté L'organisation d'une épreuve de LCA dans un examen national à visée normative semble préoccupante dans un tel contexte, ced'autant que la reproductibilité de la correction d'une épreuve de LCA n'est pas connue.

Le but de cette étude-pilote est d'apprécier la reproductibilité intercorrecteurs d'une correction d'épreuve de LCA organisée suivant les spécifications actuellement connues de cette épreuve, sans précaution méthodologique particulière, dans les conditions actuelles de formation des étudiants et de préparation des correcteurs, afin de repérer les difficultés potentielles de ce type d'épreuve.

\section{Sujets et méthodes}

\section{Etudiants}

A la faculté de médecine de Rouen, un enseignement des méthodes épidémiologiques est réalisé depuis 1998 en première année du deuxième cycle des études médicales (DCEM 1) sous forme d'un autoapprentissage des principes de méthode, suivi de séances d'enseignement dirigé de 90 minutes, utilisant des articles originaux comme supports ; cet enseignement est validé par une épreuve terminale d'analyse d'article de 30 minutes, comportant 4 à 5 questions concernant un résumé d'article 4 . Les étudiants ont été sollicités par voie d'affichage et 59 volontaires ont passé l'épreuve en septembre 2002 : 25 et 34 étaient respectivement en deuxième et troisième années du DCEM, soit respectivement 25 et $31 \%$ des inscrits des promotions concernées.

Ces étudiants ont été soumis à une épreuve dansles conditions prévues pour l'ECN, mais sans formation supplé mentaire, en particulier pour la rédaction d'un résumé.

\section{Dossier de LCA}

Un artide original a été choisi par un groupe local de cinq enseignants comme support à une épreuve de LCA : il s'agissait d'un essai randomisé bicentrique comparant deux modes de dépistage de l'hépatite $C$ en médecine générale, selon que les patients avaient reçu ou non une information préalable ${ }^{5}$. Un dossier comportant l'article (sans le résumé ni la discussion), et dix questions(T ableau 1), y comprisles réponses-type attendues (cf. annexe ।) et un résumé type (cf. annexe ॥), a été construit ${ }^{6}$; il a fait l'objet d'un travail commun des enseignants au cours de 5 réunions. Ce dossier a étéensuite validépar un groupe d'experts de LCA auprès du $\mathrm{CN} \mathrm{Cl}$ au niveau national.

\section{Epreuve}

L'épreuve a été organisée dans les conditions d'un concours : surveillance, absence d'échanges entre les étudiants, tempslimité à 3 heures, remise d'une copie rendue anonyme. Les étudiants avaient pour consigne : a) de répondre aux questions posées concernant l'article ; b) de rédiger un résumé selon les standards admis de la rédaction scientifique. La consigne précisait explicitement que le résumé devait comprendre des paragraphes respectivement dédiés aux objectifs, aux méthodes, aux résultats et à la conclusion. 


\section{Tableau 1 : \\ Q uestions utilisées pour l'épreuve d'évaluation des aptitudes à la lecture critique d'article}

Items

Points maximum

1- Pour cet essai randomisé visant à démontrer l'efficacité d'une intervention, précisez :

a) la nature précise de l'intervention qu'on cherche à évaluer

dont

b) le ou les critères de jugements utilisés

c) pourquoi y a t-il «deux unités d'analyse »

2- Cet essai justifiet-il un avis du CCPPRB ? Justifiez votre réponse.

3- Pourquoi at-on stratifié l'essai selon le mode d'exercice?

4- Les auteurs considèrent que le résultat de l'essai est négatif : quelles raisons pourraient, en théorie, expliquer ce résultat?

5- Q uel est l'effet du sexe masculin tel qu'il résulte des tableaux 4 \& 5?

6- $D$ 'après les résultats de cette étude, en quoi les toxicomanes se distinguent-ils des patients à ALAT > N vis-à-vis du dépistage de l'hépatite $\mathrm{C}$ ?

7- Les résultats obtenus dans cet essai vous paraissent-ils applicables à l'ensemble des médecins généralistes en France? Justifiez votre réponse

8- Les résultats obtenus dans cet essai vous paraissent-ils applicables à l'ensemble de la population française adulte? Justifiez votre réponse.

9- D 'après les résultats dont vous disposez, peut-on comparer la clientèle des deux groupes de médecins? Justifiez votre réponse.

10- Proposez un titre alternatif pour cet article 8

Résumé

O bjectifs

dont

M éthodes

Résultats

Conclusion

CCPPRB : Comité consultatif de protection des personnes dans la recherche biomédicale ALAT : alanine amino tranfférase 


\section{Recherche et Perspectives}

\begin{tabular}{|c|c|c|c|}
\hline \multicolumn{4}{|c|}{$\begin{array}{l}\text { Tableau } 2 \text { : } \\
\begin{array}{l}\text { Evaluation de la fidélité inter correcteur au cours de la correction de l'épreuve } \\
\text { de lecture critique d'article }\end{array} \\
\text { Coefficients de corrélation intra-classe obtenus par } 3 \text { paires de correcteurs pour } 59 \text { étudi ants selon les items, } \\
\text { avec leurs intervalles de confianceà } 95 \% \text {. }\end{array}$} \\
\hline & \multicolumn{3}{|c|}{ Paires de correcteurs } \\
\hline & $X Y$ & $\mathbf{X Z}$ & YZ \\
\hline \multicolumn{4}{|l|}{ Q uestions } \\
\hline Q1a & $0,84(0,75-0,90)$ & $0,21(-0,04-0,44)$ & $0,19(-0,06-0,42)$ \\
\hline Q 1b & $0,66(0,49-0,78)$ & $0,30(0,05-0,51)$ & $0,32(0,08-0,53)$ \\
\hline Q 1c & $0,31(0,06-0,52)$ & $0,25(0,00-0,47)$ & $0,00(-0,25-0,25)$ \\
\hline Q2 & $0,90(0,84-0,94)$ & $0,88(0,80-0,93)$ & $0,87(0,79-0,92)$ \\
\hline Q3 & $0,84(0,75-0,90)$ & $0,21(-0,04-0,44)$ & $0,19(-0,06-0,42)$ \\
\hline Q 4 & $0,52(0,31-0,68)$ & $0,38(0,14-0,58)$ & $0,33(0,08-0,54)$ \\
\hline Q5 & $0,68(0,52-0,80)$ & $0,44(0,21-0,62)$ & $0,36(0,12-0,56)$ \\
\hline Q 6 & $0,60(0,41-0,74)$ & $0,38(0,14-0,58)$ & $0,56(0,36-0,71)$ \\
\hline Q7 & $0,82(0,71-0,89)$ & $0,68(0,52-0,80)$ & $0,56(0,36-0,71)$ \\
\hline Q 8 & $0,69(0,53-0,80)$ & $0,62(0,44-0,76)$ & $0,56(0,36-0,71)$ \\
\hline Q9 & $0,74(0,60-0,84)$ & $0,34(0,10-0,55)$ & $0,10(-0,15-0,35)$ \\
\hline Q 10 & $0,63(0,44-0,76)$ & $0,60(0,40-0,74)$ & $0,45(0,22-0,63)$ \\
\hline Sous-total & $0,82(0,65-0,86)$ & $0,57(0,32-0,69)$ & $0,32(0,03-0,49)$ \\
\hline \multicolumn{4}{|l|}{ Résumé } \\
\hline $\mathrm{R} 1$ & $0,72(0,57-0,82)$ & $0,49(0,27-0,66)$ & $0,57(0,37-0,72)$ \\
\hline R2 & $0,37(0,13-0,57)$ & $0,40(0,17-0,59)$ & $0,56(0,36-0,71)$ \\
\hline R3 & $0,46(0,23-0,64)$ & $0,47(0,24-0,64)$ & $0,50(0,29-0,67)$ \\
\hline R4 & $-0,07(-0,31-0,19)$ & $0,11(-0,15-0,35)$ & $0,45(0,22-0,63)$ \\
\hline Sous-total & $0,29(0,27-0,66)$ & $0,28(0,25-0,65)$ & $0,62(0,57-0,82)$ \\
\hline Ensemble de l'épreuve & $0,70(0,52-0,80)$ & $0,72(0,55-0,81)$ & $0,48(0,26-0,65)$ \\
\hline
\end{tabular}




\section{Correction}

Trois correcteurs ( $X, Y$ et $Z$ ), enseignants à la faculté, appartenant au groupe ayant conçu le dossier, ont corrigé chacun les 59 copies de façon indépendante, à l'aide d'un barème sur 100 points, répartis entre les dix questions et le résumé (tableau 1).

\section{Analyse statistique}

Les scores portés par chaque correcteur à chaque étudiant ont fait l'objet d'une double saisie. Les scores moyens des 3 correcteurs aux mêmes copies pour les questions, d'une part, et pour le résumé, d'autre part, ont été comparés par un test $\mathrm{t}$ apparié. Les coefficients de corrélation intraclasses $^{7}(\mathrm{CCl})$ et leur intervalle de confiance à $95 \%$ (IC $95 \%$ ) ont étécalculés pour les trois couples de correcteurs pour chaquequestion, pour l'ensemble des questions, pour le résumé et enfin pour l'ensemble de l'épreuve. Les $\mathrm{CCl}$ ont été comparés par étude du recouvrement des IC $95 \%$. Le niveau de concordance a été qualifiéselon les références admises. La moyenne des $\mathrm{CCI}$ par correcteur a été calculée. La relation entre la reproductibilité des corrections et le niveau des copies a été testée par un coefficient de corrélation entre la moyenne des $\mathrm{CCl}$ et celle des scores obtenus par les étudiants.

\section{Résultats}

Le scores moyen ( \pm écart type) sur 20 donné par les 3 correcteurs à l'ensemble de l'épreuve aux 59 étudiants était de 8,7 $( \pm 2,0)$ avec une dispersion modérée (minimum 4,5 ; maximum : 14,6). Le scores moyen était de $8,2( \pm 2,2)$ pour les questions et de 9,9 $( \pm 2,6)$ pour le résumé. L'écart moyen entre les scores du résumé et des questions de chaque étudiant était de $1,7( \pm 2,6)$, écart supérieur à zéro $\left(p<10^{-6}\right)$.

Les $\mathrm{CCI}$ des questions, du résumé et de l'ensemble des épreuves figurent dans le Tableau 2 pour les 3 paires de correcteurs. Pour l'ensemble de l'épreuve, les résultats variaient de 0,48 (IC $95 \%: 0,26$ - 0,65) pour la paire YZ à 0,72 (IC $95 \%: 0,55$ - 0,81) pour la paire XZ.

Sur les $48 \mathrm{CCl}$ (16 items pour 3 paires), aucun n'était très bon, 8 étaient bons, 14 modérément bons, 16 médiocres et 10 mauvais selon les qualificatifs usuels.

La correction des questions était plus reproductible que celle des résumés pour 2 des 3 paires de correcteurs.

La reproductibilité (moyenne des $\mathrm{CCI}$ des trois paires de correcteurs) n'était pas significativement liée avec le score moyen obtenu par les étudiants $(r=-0,35)$.

\section{Discussion}

Ce travail pilote comporte des limites notables : les étudiants étaient volontaires, issus d'une seule faculté et peu nombreux ; ils n'étaient concernés par l'ECN que 2 à 3 ans plus tard, délai permettant des apprentissages complémentaires; leur habilitée ${ }^{8}$ telle qu'ellea étéévaluée dans cetravail, ne correspond pasà celle correspondant à la date prévue pour eux pour cet examen. L'absence d'enjeu pour eux modifiait les conditions de l'épreuve et peut-être leur comportement. L'analyse ne portait que sur un seul article, corrigé par des enseignants d'une seule faculté, la plupart n'ayant pas une expérience notable de corrections d'épreuves de LCA.

Ces étudiants n'avaient pas encore fait l'objet d'une préparation spécifique de la LCA en vue de l'ECN, mais ils avaient reçu une formation méthodologique en DCEM 1 , formation sappuyant en bonne partie sur l'analyse d'articles scientifiques. Toutefois, cette situation correspond probablement à celle d'autres facultés en France actuellement ; elle est donc opportune pour juger des performances qui pourrai ent être associées à une telle épreuve, sans précautions particulières.

D ans ces conditions, ce groupe d'étudiants de $2^{e}$ cycle n'a pas obtenu la moyenne à cette épreuve de LCA respectant les conditions définies par le $\mathrm{CNCl}$; l'épreuve est donc apparue difficile. Le score moyen obtenu au résumé était paradoxalement meilleur que pour les questions, alors que les étudiants n'avaient pas été formés spécifiquement à cet exercice?.

L'épreuve est également apparue peu discriminante, d'après la dispersion des scores. En outre, sa correction a été difficile, comme le suggère la faible reproductibilité inter-juges. La reproductibilité du processus de correction pouvait être influencée par l'épreuve ellemême, par les items qui la composaient, par les étudiants ou par les évaluateurs. La valeur moyenne des $\mathrm{CCI}$ des questions et du résumé étaient proches, correspondant à un accord inter-juges modéré; ce résultat médiocre n'était pas le fait de l'une ou l'autre des deux parties del'épreuve. La reproductibilité de la correction variait fortement selon les items : elle était médiocre ou mauvaise pour plus de la moitié d'entre eux, mais surtout la dispersion des $\mathrm{CCl}$ était importante (extrêmes: - 0,07 à 0,90).

Certaines difficultés de correction imprévues ont été rencontrées pour certains items, mettant en difficultéles trois correcteurs ( $Q 1 c$ et conclusion du résumé) ; on peut s'interroger sur le libellé de telles questions, voire leur contenu réel. 


\section{Recherche et Perspectives}

II apparât légitime de n'utiliser en situation de concours que des questions ayant été testées en conditions réelles, non seulement en terme de difficulté de réponse, mais encore en terme de fiabilité de correction.

La reproductibilité de la correction n'était pas liée avec les scores des étudiants, bien que ce résultat n'ait éé établi que sur un effectif restreint. Ce point suggère que cette reproductibilité n'était pas dépendante du niveau des étudiants. Cependant, les étudiants n'avaient probablement pas toutes les habiletés requises dans le cadre de référence ${ }^{8}$, comme celle d'évaluer la pertinence de l'article, la validité des résultats, leur utilité pratique. Ils n'avaient eu aucun apprentissage de la rédaction d'un résumé, alors que l'entrainement améliore les résultats ${ }^{10}$.

La médiocre reproductibilité des corrections peut enfin provenir des correcteurs eux-mêmes, bien que ceux-ci aient directement contribuéà l'élaboration du dossier, en particulier des réponses-type ; ce point suggère l'importance d'utiliser une grille de correction pour ce type d'épreuve; il serait intéressant de vérifier qu'une telle grille permettrait bien de réduire la variabilité inter-correcteur.

L'épreuve de LCA, telle qu'elle est envisagée actuellement, est restreinte aux articles originaux ; cependant, le résumé, dont ils ne devraient pas disposer dans les conditions pré vues pour l'ECN, joue un rôle important pour la formation des médecins en exercice; en outre, ces derniers sont fréquemment confrontés à d'autres types de publication, comme des revues systématiques ou des recommandations de bonne pratique; inversement, la rédaction d'un résumé en vu d'une publication n'est pas une activité habituelle pour un médecin en exercice; les habiletés jugées nécessaires pour la pratique professionnelle ultérieure ne sont pas donc appréciées sur des bases très réalistes ; cependant les avantages et inconvénients des différents constituants prévus pour l'ECN (dossiers, LCA) ont été peu étudiés. Plus généralement, d'autres types d'épreuves que la LCA peuvent être envisagés pour pallier les inconvénients des questions rédactionnelles à développements longs ${ }^{11}$.

\section{Conclusion}

Cette étude pilote suggère la difficulté d'une épreuve de LCA pour les étudiants, la médiocrité de sa reproductibilité inter correcteurs et souligne l'importance de précautions à prendre pour la correction, comme l'utilisation d'une grille de correction et une correction multiple.

Si une telle épreuve doit classer plus de 5000 candidats au niveau national, une formation spécifique des étudiants et des enseignants, une préparation et un test des épreuves et de leur correction par un travail transversal au sein de toutes les facultés semble indispensable dans un souci d'équité et d'éthique pédagogique.

\section{Remerciements}

Les auteurs remercient les 59 étudiants qui se sont prêtés au tet, ains que $M$. Richard M ederos qui a revu la version angl aise du résumé.

\section{Contributions}

Francis Roussel et Pierre Czernichow ont participé à l'élaboration du dossier utilisé pour l'étude, corrigé les épreuves, réalisé l'analyse des données de l'étude et rédigé une première version du manuscrit ; ils ont effectué la révision du manuscrit à partir des critiques des lecteurs. Alain Lavoinne, Jean-François Lemeland ont participé à l'élaboration du dossier utilisé pour l'étude, et contribué à la relecture et l'élaboration de la version du manuscrit qui a été soumise à la revue. Jean-Paul Fillastre a participé à l'élaboration du dossier utilisé pour l'étude, corrigé les épreuves, et contribuéà la relecture et l'élaboration de la version du manuscrit qui a été soumise à la revue. 


\section{Annexel: \\ Réponses type utilisées pour la correction des 59 copies par les correcteurs}

1a. Information des patients par affiche et dépliants en salle d'attente.

1b. Pourcentage demédecins prescripteurs, pourcentage de sérologies prescrites, pourcentages des sérologies demandées par les malades, pourcentages des sérologies effectuées, pourcentages des sérologies positives.

1c. Parce qu'il sagit d'un essai avec randomisation de groupes (clientèles de médecins) et non de patients : I'analyse porte sur les médecins (prescripteurs ou pas) et sur les patients auxquels une sérol ogie a été prescrite.

2. N on. Pas d'intervention faisant courir un risque particulier aux patients; I'offre d'une sérologiefait partie des soins de routine. II existe un bénéfice direct.

3. On a pensé que le mode d'exercice pouvait influencer le critère de jugement. On a pensé que le mode d'exercice pouvait influencer le recrutement. $0 \mathrm{n}$ a voulu s'assurer qu'il ne perturberait pas l'analyse (deux tirages au sort sont effectués : un dans chaque strate, un chez les médecins exerçant seul, un autre chez ceux exerçant en groupe). O u bien : le mode d'exercice a été considéré comme un facteur de confusion possible.

4. Effectifs insuffisants (défaut de puissance) ; biais dans la sélection des patients (répartition différente des groupes à risque dans les clientèles) ; comportement particulier des toxicomanes (12 sérologies positives mathématique ment manquantes pour 25 trouvées au total) ; biais dans la mesure des prescriptions des sérologies (données manquantes, perdus de vue) ; inefficacité de l'intervention si les autres rai sons sont éliminées.

5. Les hommes réalisent 2 fois plus souvent la sérologie prescrite que les femmes, tous facteurs égaux par ailleurs, al ors qu'il n'y a pas de lien entre le sexe et la positivité de la sérologie.

6. Les toxicomanes réal isent moins souvent la sérologie $(O R=0,04)$ que les patientsALAT $>N$, tousfacteurs égaux par ailleurs, alors qu'ils ont un risque supérieur d'être positifs $(O R=19,8)$.

7. N on. Les médecins sont volontaires, donc non représentatifs des 2 régions concernées. Leur fréquence est d'ailleurs très différente. Ils proviennent de 2 régions particulières.

8. N on. II sagit de consultants de médecins généralistes, donc il existe une sélection par le recours aux soins. Ils proviennent de 2 régions particulières.

9. Non. Seules les caractéristiques des patients ayant fait l'objet d'une prescription de sérologie ont fait l'objet d'un recueil de données : on peut donc comparer : les caractéristiques des médecins, les caractéristiques des malades ayant eu une prescription de sérologie ; mais on ne peut pas comparer les caractéristiques de l'ensemble des malades.

10. Efficacité d'une information en salle d'attente sur le dépistage de l'hépatite C : essai randomisé chez 184 médecins généralistes du D oubs et de l'lle de France. 


\section{Recherche et Perspectives}

\section{Annexe II : \\ Résumé utilisé pour la correction des 59 copies par les correcteurs ( 248 mots)}

O bjectif : Evaluer l'efficacité d'une information par affiche et dépliants en salle d'attente de médecine générale sur le dépistage de l'hépatite $C$.

M éthodes : 184 médecins volontaires du D oubs $(n=119)$ et d'Ile de France $(n=65)$ ont été répartis par tirage au sort stratifié sur le mode d'exercice (isolé ou en groupe) : groupe $1(n=90)$ : recherche de facteur de risque d'hépatite $C$ et offre éventuelle d'une sérologie sur l'initiative du médecin ; groupe $2(n=94)$ : id et information préalable sur l'hé patite $C$ des patients (affiche et dépliants) en salle d'attente. Le pourcentage de prescripteurs et le nombre de sérologies ont été comparés respectivement par les tests du khi-carré et de M ann-Whitney. Enfin les facteurs associés à la réal isation des sérologies prescrites, et à la séropositivité ont été recherchés par régression logistique.

Résultats : En 15 mois 55 médecins du groupe 1 (61\%) et 48 du groupe 2 (51\%) (non significatif) ont prescrit 323 et 294 sérologies respectivement (non significatif). Les patients du groupe 2 ayant eu une sérologie étaient plus âgés; l leurs facteurs de risque étaient similai res mais plus nombreux. La réalisation des sérologies prescrites était plus fréquente chez les 60 ans et plus, et les hommes, moins fréquente chez les toxicomanes, et ceux ayant un tatouage. La séropositivité n'était liée qu'aux patients toxicomanes. Son contrôle était plus fréquemment demandé par les patients informés, mais pas plus fréquemment réalisé et pas plus fréquemment positif.

Conclusion : L'information n'a pas amélioré le rendement du dépistage de l'hépatite C.

\section{Références}

1. Fillastre JP, Colin R. Analyse critique ou lecture critique des artides médicaux. Quelle cible choisir pour l'enségnement et l'évaluation? Pédagogie M édicale 2001 ; 2 : 197-198.

2. Conseil scientifique du centrenational du concours d'internat. Epreaveécrite de lecture d'artide 2003 [on-line]. Disponible sur : http://www.ond. univ-paris5.fr/medednelCritiqueArtidedoc

3. Jouquan J. L'évaluation des apprentissages des étudiants en formation médicale initiale. Pédagogie Médicale $2002 ; 3: 38-52$.

4. Tavolacci M P, Ladner J, Kerdiles M, H allais C, Josset V, $M$ erle $V$ et al. Un nouveau dispostif de formation en santé publique danslesétudes médicales: miseen place et mesures d'impact. Pédagogie M édicale $2005 ; 6$ : 15-24.

5. Roudot-Thoraval $F, M$ onnet $E, M$ ercet $P$, Bastie $A$, Dhumeaux $D$, M iguet JP. Stratégies de dépistage de I'hépatite virale $C$ en $M$ édecine générale. Résultats d'un essai randomiǽ bicentrique Gastroenterol Clin Biol. $2000 ; 24: 1037-1041$.
6. Beaucage C, Viger Y. Epidémiologie appliquée : une initiation à la lecture critique de la littérature en sciences de la santé Boucherville (QC) : Gaëan M orin éditeur, 1996.

7. Fermanian J. Mesure de l'accord entre deux juges cas quantitatif. Rev Epidem et santé publ 1984 ; 32 : 408-413.

8. Coté L, Turgeon L. Comment lire de façon critique les articles de recherche qualitative en médecine? Pédagogie M édicale $2002 ; 3$ : 81-90.

9. Audet $N$, Leclère H. Les habilités requi ses pour la lecture critique en médecine : un cadre de référence issu d'une recension des écrits. Pédagogie M édicale 2001 ; 2 : 206-212.

10. Taylor R, Reeves B, Ewings P, Binnes S, Keast J, M ears R. A systematic review of the effectiveness of critical appraisal killstraining for dinicians M ed Educ 2000 ; $34: 120-125$.

11. Stern DT, Linzer M, O'Sullivan PS, Weld $L$. Evaluating medical residents literatureappraisal skills. Acad M ed $1995 ; 70$ : 152-154.

M anuscrit reçu le 11 mars 2004 ; commentaires éditoriaux formulés aux auteurs le 5 octobre 2004 ; accepté pour publication le 21 décembre 2004. 